\title{
Temperature Effects on Ion-Desorption Dynamics from Solid Ne by Low-Energy Ion Impacts
}

\author{
S. Jinno ${ }^{a}$, T. Isozaki ${ }^{b}$, I. Naemura ${ }^{b}$, T. Koizumi ${ }^{a, b}$, T. Hirayama ${ }^{a, b}$ \\ ${ }^{a}$ Research Center for Measurements in Advanced Science (RCMAS), Rikkyo University \\ Nishi-Ikebukuro, Toshima-ku, Tokyo 171-8501, Japan \\ ${ }^{b}$ Department of Physics, Rikkyo University, Nishi-Ikebukuro, Toshima-ku, Tokyo \\ 171-8501, Japan
}

\begin{abstract}
Interactions between low-energy ions and solid rare gases have been investigated by observing desorbed ions. When low-energy ions were injected into solid Ne, we found that the kinetic energy of desorbed cluster ions significantly increased with irradiation time for a film thickness of several hundred monolayers. This result can be explained by charge-up (i.e., electronic holes) in the solid Ne. The kinetic energy of the desorbed ions is considered to be proportional to the number of holes created in the solid by the ion impacts. The solid's temperature effects on the desorbed-ion kinetic energy can be understood as a dependence on the diffusion rate of the holes. The temperature was controlled between 4.7 and 7.0 K. The activation energy of hole-hopping transport is estimated at $0.7 \mathrm{meV}$ based on the slope of Arrhenius plots.
\end{abstract}

\section{Introduction}

When charged particles are injected into insulators or semiconductors, charge-up often occurs by emission of secondary electrons. The charge-up phenomenon can cause difficulties in, for example, obtaining accurate measurements of secondary and photo-electron yields in a scanning electron microscopy (SEM) and a X-ray photoelectron spectroscopy (XPS), and energy analysis of desorbed ions and Auger electrons in various surface analyses. Charge-up causes damage to the oxide film of transistors because of discharges in plasma-etching processes.

On the other hand, a number of recent studies have taken advantage of charge-up for applications in beam-guiding systems. For example, a beam 
can be transported to the desired position through a glass capillary by means of the repulsive force between the beam and the charge-up that occurs on the surface of the capillary $[1,2,3]$.

Consequently, it is important that the charge-up phenomenon is studied in detail in a simple system. Kinetically induced sputtering of rare-gas solids by low-energy ion impacts has been investigated for several decades $[4,5$, $6,7,8,9,10]$. However, charge-up in rare-gas solids, which is the simplest insulating material, has not been studied in detail. In this paper, we discuss the temperature effect of charge-up in solid Ne caused by low-energy ion impacts. We found that the amount of charge-up can be determined by measuring the kinetic-energy distributions of desorbed ions, which shift to higher energy with increasing irradiation time. Relaxation of charge-up is observed to depend on the temperature of the solid. This can be understood in terms of diffusion or hopping transport of electronic holes. To identify the conditions leading to a reduction of charge-up, and the methods by which to achieve this, will contribute to more precise measurements in terms of, for example, energy analysis of desorbed ions and Auger electron spectroscopy.

\section{Experimental method}

Figure 1 shows a schematic view of the main chamber. $\mathrm{Ar}^{+}$ions are generated by an electron-cyclotron-resonance ion source (NANOGAN). Extracted ions are mass-selected by an analyzing magnet and transported to the surface of solid Ne at the center of the main chamber. A polycrystalline $\mathrm{Cu}$ substrate is cooled to $4.7 \mathrm{~K}$ by a mechanical cryostat. The background pressure is $3 \times 10^{-8} \mathrm{~Pa}$. The solid $\mathrm{Ne}$ is condensed onto the $\mathrm{Cu}$ disk by filling the chamber with gaseous $\mathrm{Ne}$ to a pressure of approximately $10^{-4} \mathrm{~Pa}$. The film thickness was estimated from the exposure assuming the condensation coefficient to be unity.

The incident angle of the beam is $60^{\circ}$ from the normal direction of the sample surface, and the detector for desorbed ion species is installed in the direction normal to the sample surface. The desorbed ions are mass-selected by a quadrupole mass spectrometer (QMS; MSQ-400, ULVAC) and detected by a channeltron detector in pulse-counting mode. A bias potential $\left(V_{\mathrm{b}}\right)$ of $+15 \mathrm{~V}$ is applied to the sample substrate to accelerate the desorbed ions. The kinetic-energy distribution of the desorbed ions is measured by sweeping the retarding potential $\left(V_{\mathrm{r}}\right)$ to a four-grid-type energy analyzer mounted in front of the QMS. Ions with initial kinetic energy greater than $e\left(V_{\mathrm{r}}-V_{\mathrm{b}}\right)$ can pass 
through the energy analyzer and are counted. The desorbed-ion transmission efficiency may slightly depend on the retarding potential, however, a relative comparison of the mean kinetic energy is possible using this method.

Since the desorption yield, most of which are neutral particles, is very large for solid $\mathrm{Ne}[11,12]$, the incident ion-beam current was adjusted to less than $3 \mathrm{nA}$ to minimize changes in sample thickness during the measurements. The temperature of the sample substrate was maintained at a predetermined value using a heater with a temperature controller. After the beam had irradiated the sample for several minutes and the desorbed-ion kinetic energy had become saturated, the measurement was started. The sample was evaporated and redeposited after each measurement.

The ion range of $1.8 \mathrm{keV} \mathrm{Ar}^{+}$ions into the solid Ne was approximately $5 \mathrm{~nm}$ according to calculation by SRIM2008 [13]. Since this corresponds to 20 monolayers (MLs) of solid Ne, the influence of the substrate was negligible in terms of sputtering and hole formation in our experiments. We did not correct for contact potential.

\section{Results and discussion}

Figure 2 shows a mass spectrum of desorbed cluster ions from solid $\mathrm{Ne}$ with a thickness of $2000 \mathrm{MLs}$ at $4.9 \mathrm{~K}$ by $1.8 \mathrm{keV} \mathrm{Ar}^{+}$-ion impact. The main desorbing ion species are the cluster ions $\mathrm{Ne}_{n}^{+}$( $n$ : cluster size), and the largest peak is the Ne dimer ion. The peak at $m / q=28$ is identified as being due to $\mathrm{CO}^{+}$and/or $\mathrm{N}_{2}^{+}$impurities. The small peaks on the right-hand side of each detected $\mathrm{Ne}_{n}^{+}$cluster peak show clusters that include neon isotopes $\left({ }^{21} \mathrm{Ne}\right.$ and $\left.{ }^{22} \mathrm{Ne}\right)$, whose natural abundance is $0.27 \%$ and $9.25 \%$, respectively. The combination including neon isotopes increases with larger cluster sizes.

Figure 3 shows normalized integrated kinetic-energy spectra of desorbed $\mathrm{Ne}_{n}^{+}(n=1-9)$ ions from the surface of solid Ne $(2000 \mathrm{MLs})$ at $4.9 \mathrm{~K}$ and impacted by $1.8 \mathrm{keV} \mathrm{Ar}^{+}$ions. The kinetic energies of $\mathrm{Ne}_{n}^{+}(n=1-3)$ decrease with size, while those of $\mathrm{Ne}_{n}^{+}(n \geq 4)$ ions are largely independent of size. A detailed discussion of the process of Ne-cluster-ion formation and the dependence of kinetic energy on cluster size is given elsewhere [14].

Figure 4 shows the temperature dependence of mean kinetic energies of $\mathrm{Ne}_{2}^{+}$desorbed from solid Ne with a thickness from 200 to 1000 MLs at a sample temperature of $4.7-7.0 \mathrm{~K}$. $\mathrm{Ne}_{2}^{+}$is selected because of its large yield. The mean kinetic energy was defined as $e\left(V_{\mathrm{r}}^{\prime}-V_{\mathrm{b}}\right)$, where $V_{\mathrm{r}}^{\prime}$ is the retarding potential where the ion intensity has half the value of that at $V_{\mathrm{r}}-V_{\mathrm{b}}=0$. 
The mean kinetic energy increases with thickness and reaches approximately $10 \mathrm{eV}$ at low temperatures. This extraordinarily high energy may be the result of charge accumulation in solid Ne. This charge-up phenomenon is mostly caused by emission of secondary electrons generated by the ion impacts. In fact, the desorption energy is known to be below $2 \mathrm{eV}$, as measured for 120 MLs solid Ne, for which no charge-up effect is expected [14]. The kinetic energy of $\mathrm{Ar}_{2}^{+}$photodesorbed from solid $\mathrm{Ar}$ was reported to be less than $1 \mathrm{eV}$ [15]. This low kinetic energy was successfully explained in the framework of the desorption induced by electronic transitions (DIET). This indicates that high kinetic energy found in the present results can not be explained by purely electronic sputtering process.

Figure 5 shows the mean kinetic energy of $\mathrm{Ne}_{2}^{+}$desorbed from 2000-MLthick solid Ne at a sample temperature of $4.9-5.6 \mathrm{~K}$. The results provide information on the transport of holes in solid rare gases. The kinetic energy is assumed to be proportional to the number of holes. The accumulated charge in the solid is determined by the balance between hole formation by ion impacts and the decrease in the number of holes due to their diffusion into the metal substrate; the latter should depend on the temperature. The activation energy of hole-hopping transport is estimated to be $0.7 \mathrm{meV}$ from the slope of the Arrhenius plots shown in Fig. 5.

Drift mobilities of the holes in rare-gas solids at a temperature of $18-25 \mathrm{~K}$ were measured previously [16]. The activation energy was reported to be $24 \mathrm{meV}$, much higher than the present result. The likely reason for this discrepancy is that they measure at over a triple-point temperature of $13.57 \mathrm{~K}$ of $\mathrm{Ne}[17]$.

\section{Conclusions}

We measured the temperature dependence of the kinetic energy of desorbed ions from solid Ne by $1.8 \mathrm{keV} \mathrm{Ar}^{+}$impacts. The kinetic energy changes by more than $1 \mathrm{eV}$ following a temperature change of less than $1 \mathrm{~K}$. The rea-

son for this change is a temperature effect related to the diffusion rate of the holes. The activation energy is estimated to be $0.7 \mathrm{meV}$.

\section{Acknowledgments}

This work was supported in part by a High-Tech Research Center Project for Private Universities, a matching-fund subsidy from MEXT (Ministry of 
Education, Culture, Sports, Science, and Technology of Japan), and by the Rikkyo University Special Fund for Research.

\section{References}

[1] N. Stolterfoht, J. H. Bremer, V. Hoffmann, R. Hellhammer, D. Fink, A. Petrov, B. Sulik, Phys. Rev. Lett. 88 (2002) 133201.

[2] T. Nebiki, T. Yamamoto, T. Narusawa, M. B. H. Breese, E. J. Teo, F. Watt, J. Vac. Sci. Technol. A 21 (2003) 1671.

[3] T. Ikeda, Y. Kanai, T. M. Kojima, Y. Iwai, T. Kambara, Y. Yamazaki, M. Hoshino, T. Nebiki, T. Narusawa, Appl. Phys. Lett. 89 (2006) 163502.

[4] R. G. Orth, H. T. Jonkman, D. H. Powell, J. Michl, J. Am. Chem. Soc. 103 (1981) 6026.

[5] D. E. David, T. F. Magnera, R. Tian, D. Stulik, J. Michl, Nucl. Instrum. Meth. Phys. Res. B 14 (1986) 378.

[6] O. Ellegaard, J. Schou, H. Sørensen, Nucl. Instrum. Meth. Phys. Res. B 13 (1986) 567.

[7] J. Schou, O. Ellegaard, H. Sørensen, R. Pedrys, Nucl. Instrum. Meth. Phys. Res. B 33 (1988) 808.

[8] V. Balaji, D. E. David, T. F. Magnera, J. Michl, H. M. Urbassek, Nucl. Instrum. Meth. Phys. Res. B 46 (1990) 435.

[9] R. E. Johnson, J. Schou, Mat. Fys. Medd. Da. Vid. Selsk. 43 (1993) 404.

[10] R. Pedrys, B. Warczak, P. Leskiewicz, J. Schou, O. Ellegaard, Nucl. Instrum. Meth. Phys. Res. B 157 (1999) 121.

[11] T. Hirayama, I. Arakawa, J. Phys. Cond. Matt. 18 (2006) S1563.

[12] S. Fujita, K. Fukai, T. Tachibana, T. Koizumi, T. Hirayama, J. Phys. Conf. Ser. 163 (2009) 012083.

[13] Ziegler J F 2008 Computer codes SRIM-2008, http://www.srim.org/. 
[14] T. Tachibana, K. Fukai, T. Koizumi, T. Hirayama, J. Phys. Cond. Matt. 22 (2010) 475002.

[15] G. Dujardin, L. Philippe, M. Rose, T. Hirayama, M. J. Ramage, G. Comtet, L. Hellener, Appl. Phys. A 66 (1998) 527.

[16] P. G. L. Comber, R. J. Loveland, W. E. Spear, Phys. Rev. B 11 (1975) 3124.

[17] R. E. Rapp, E. P. de Souza, E. Lerner, Phys. Rev. B 24 (1981) 2196. 


\section{List of figure captions}

Figure 1. Schematic of the main chamber (top view). BAG: BayardAlpert ionization gauge, TMP: Tandem turbo molecular pump, FC: Movable Faraday cup, RGS/Cu: Rare-gas solid and $\mathrm{Cu}$ substrate. Inset shows a analyzer system of desorbed ions. $V_{\mathrm{b}}$ and $V_{\mathrm{r}}$ indicate a bias potential and a retarding potential, respectively.

Figure 2. Mass spectrum of desorbed $\mathrm{Ne}_{n}^{+}$from 2000-ML-thick solid $\mathrm{Ne}$ by $1.8 \mathrm{keV} \mathrm{Ar}^{+}$-ion impact.

Figure 3. Normalized integrated kinetic-energy distributions of desorbed $\mathrm{Ne}_{n}^{+}(n=1-9)$ from the surface of solid Ne (2000 MLs) at $4.9 \mathrm{~K}$ impacted by $1.8 \mathrm{keV} \mathrm{Ar}^{+}$ions.

Figure 4. Mean kinetic energy of the $\mathrm{Ne}_{2}^{+}$desorbed from solid Ne from 200 to 1000 MLs at $4.7-7.0 \mathrm{~K}$.

Figure 5. Mean kinetic energy of desorbed $\mathrm{Ne}_{2}^{+}$as a function of the temperature of the solid Ne. 


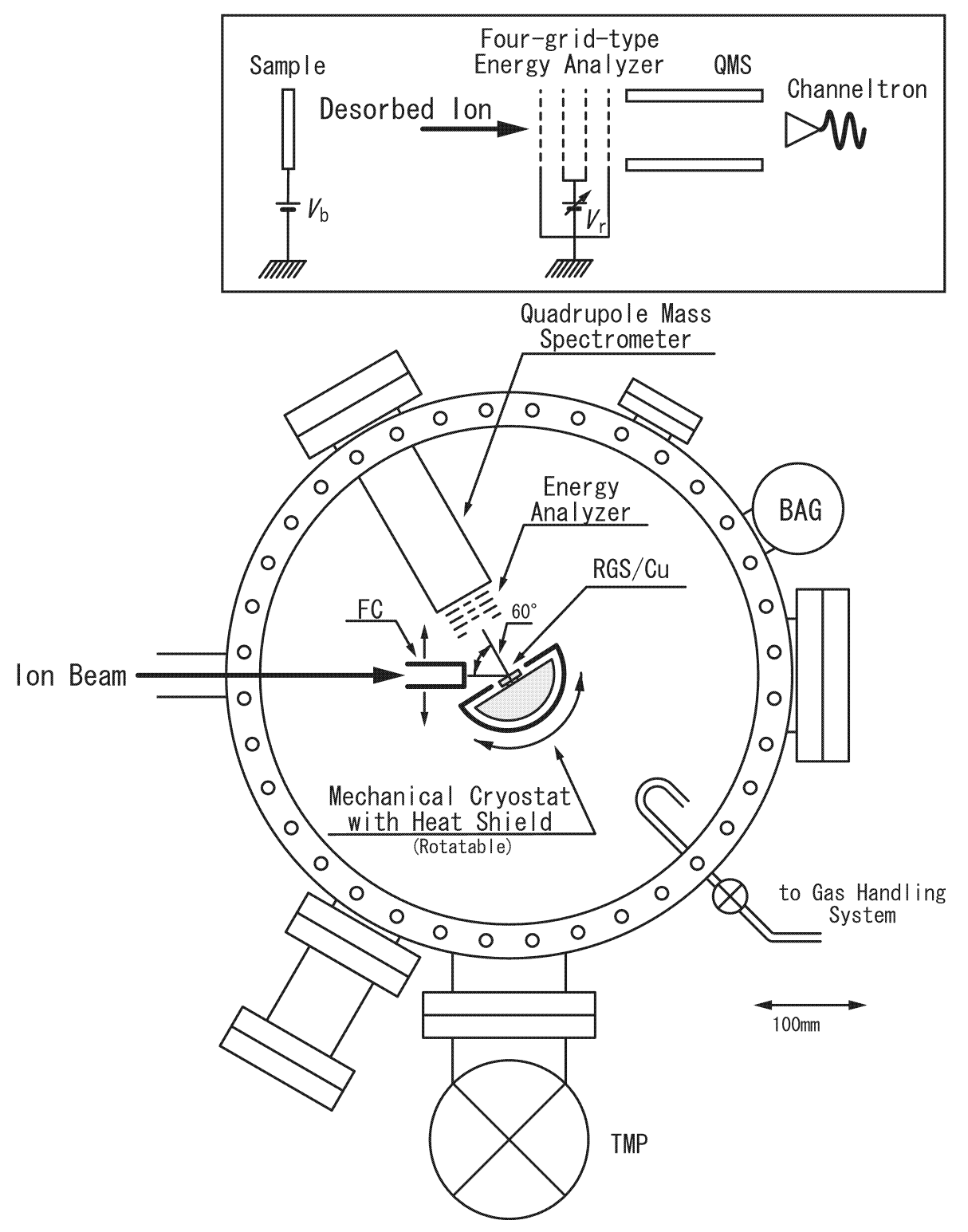

JINNO FIGURE. 1 


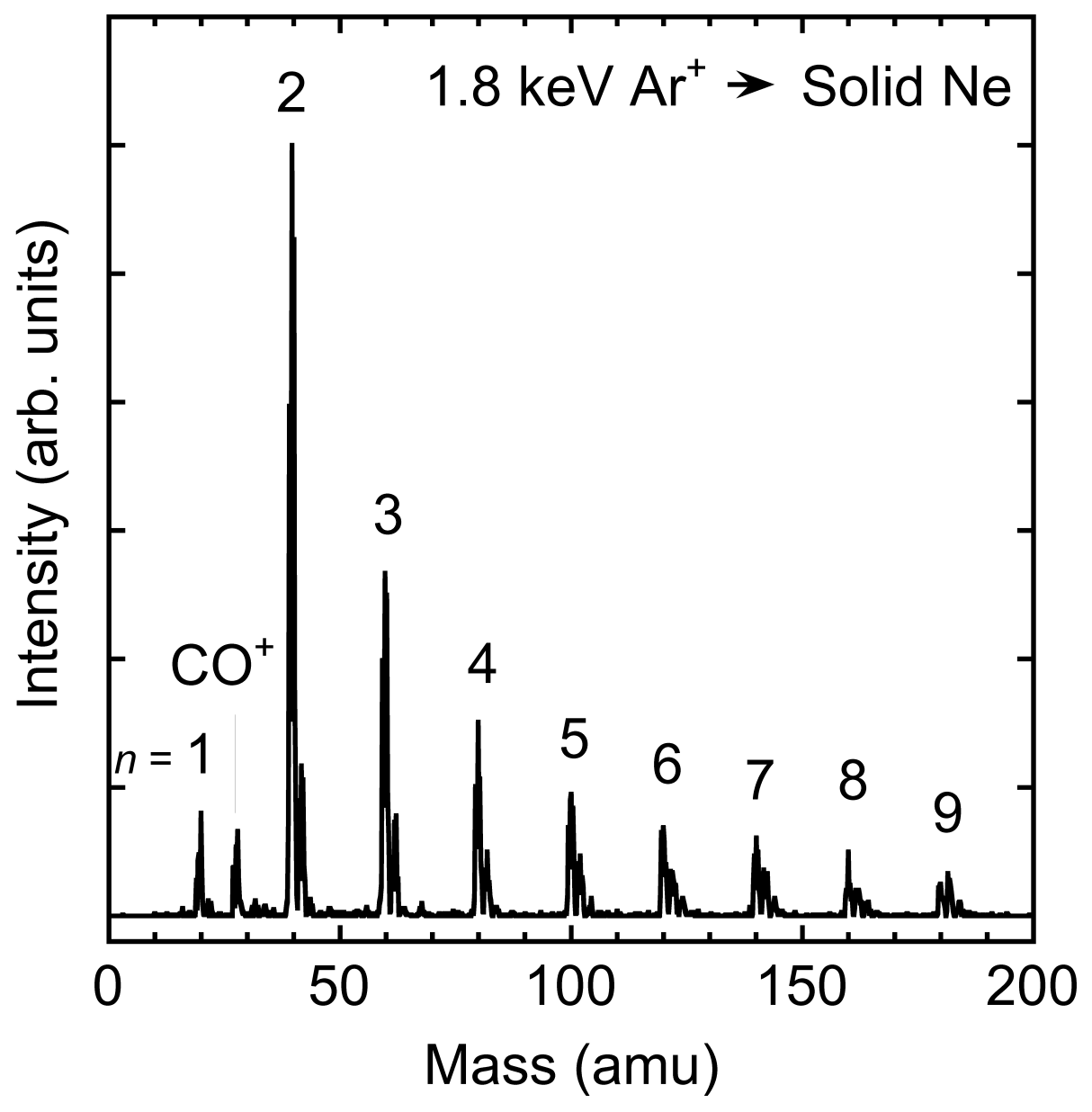

JINNO FIGURE. 2 


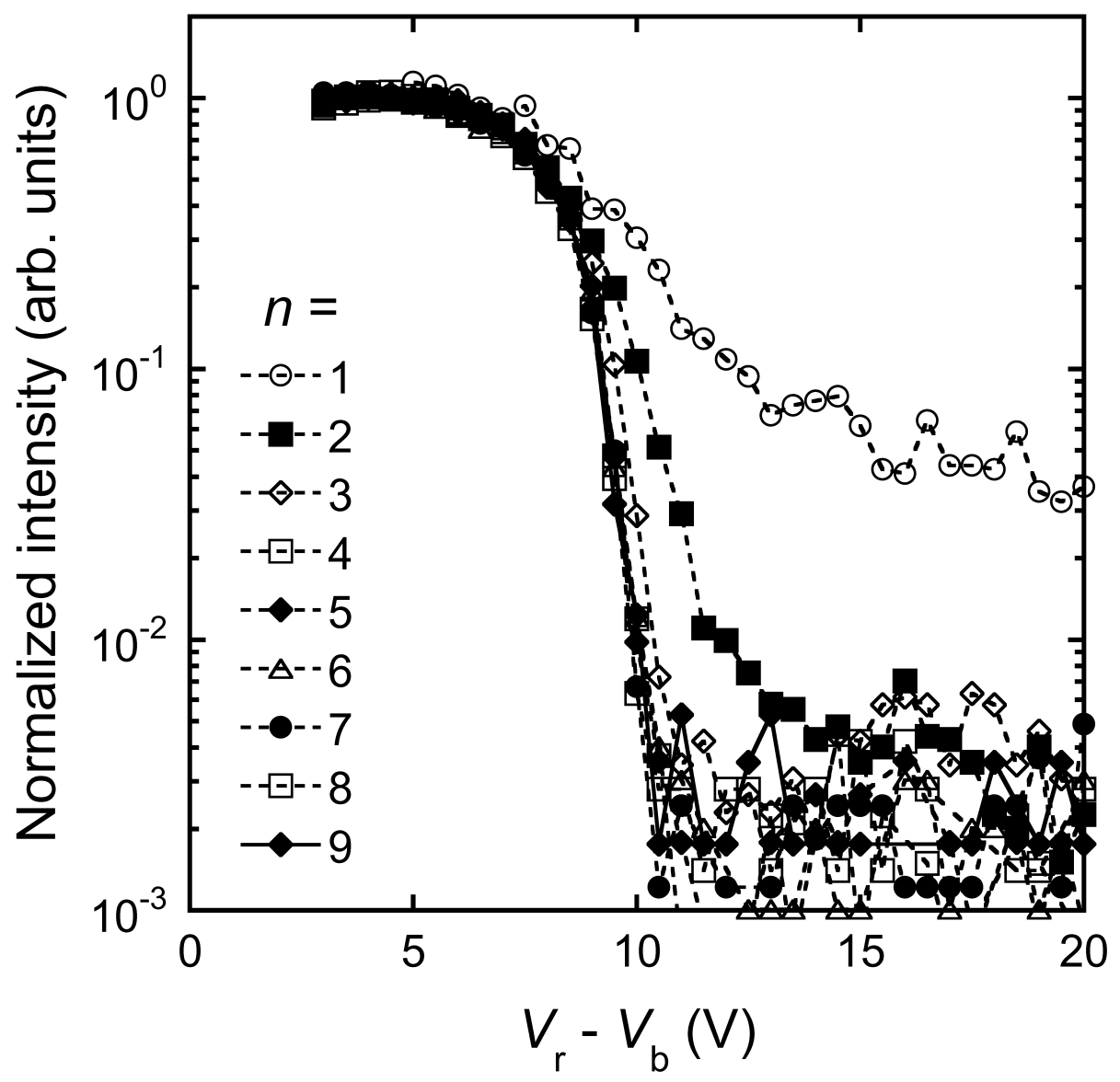

JINNO FIGURE. 3 


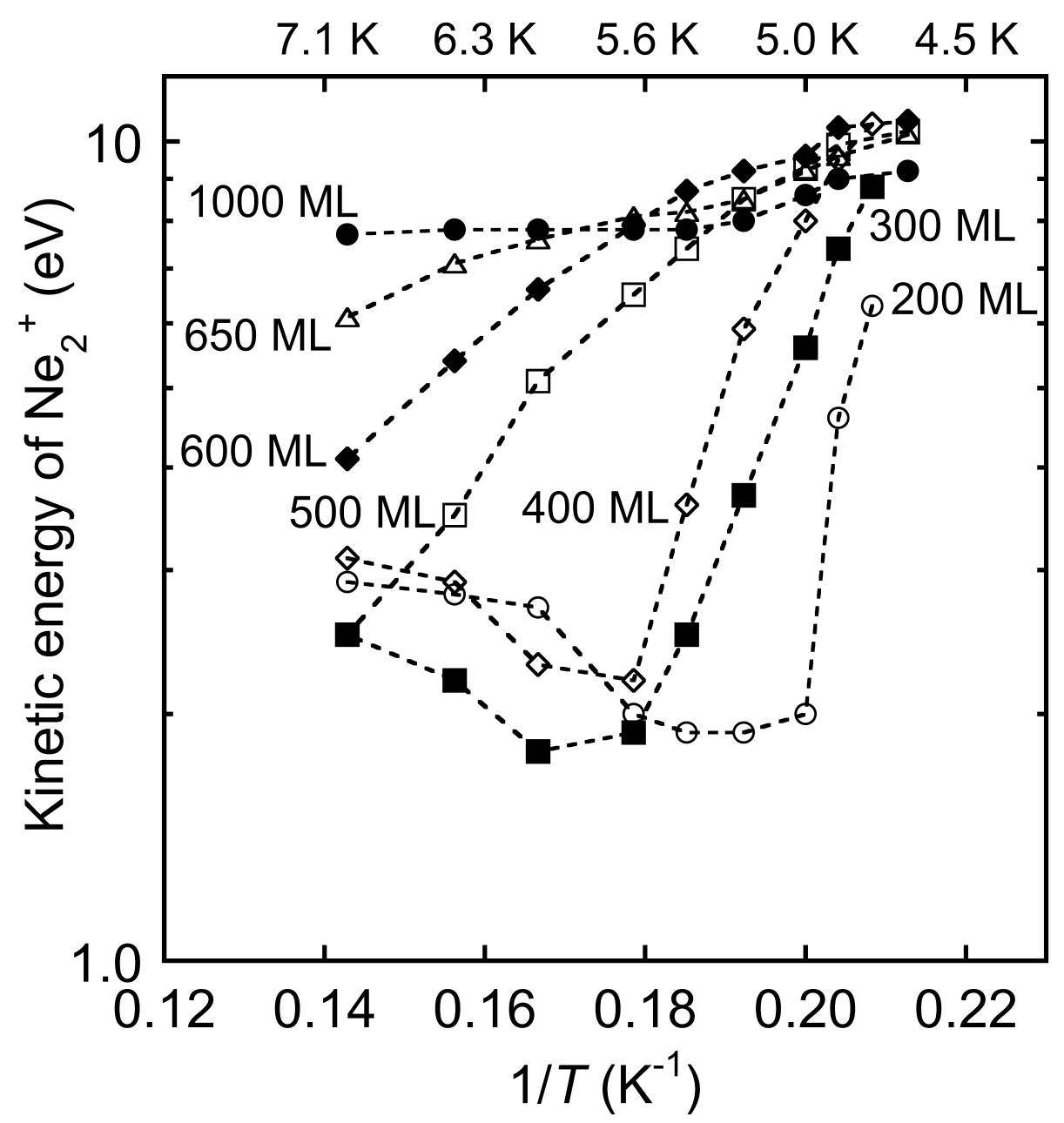

JINNO FIGURE. 4 


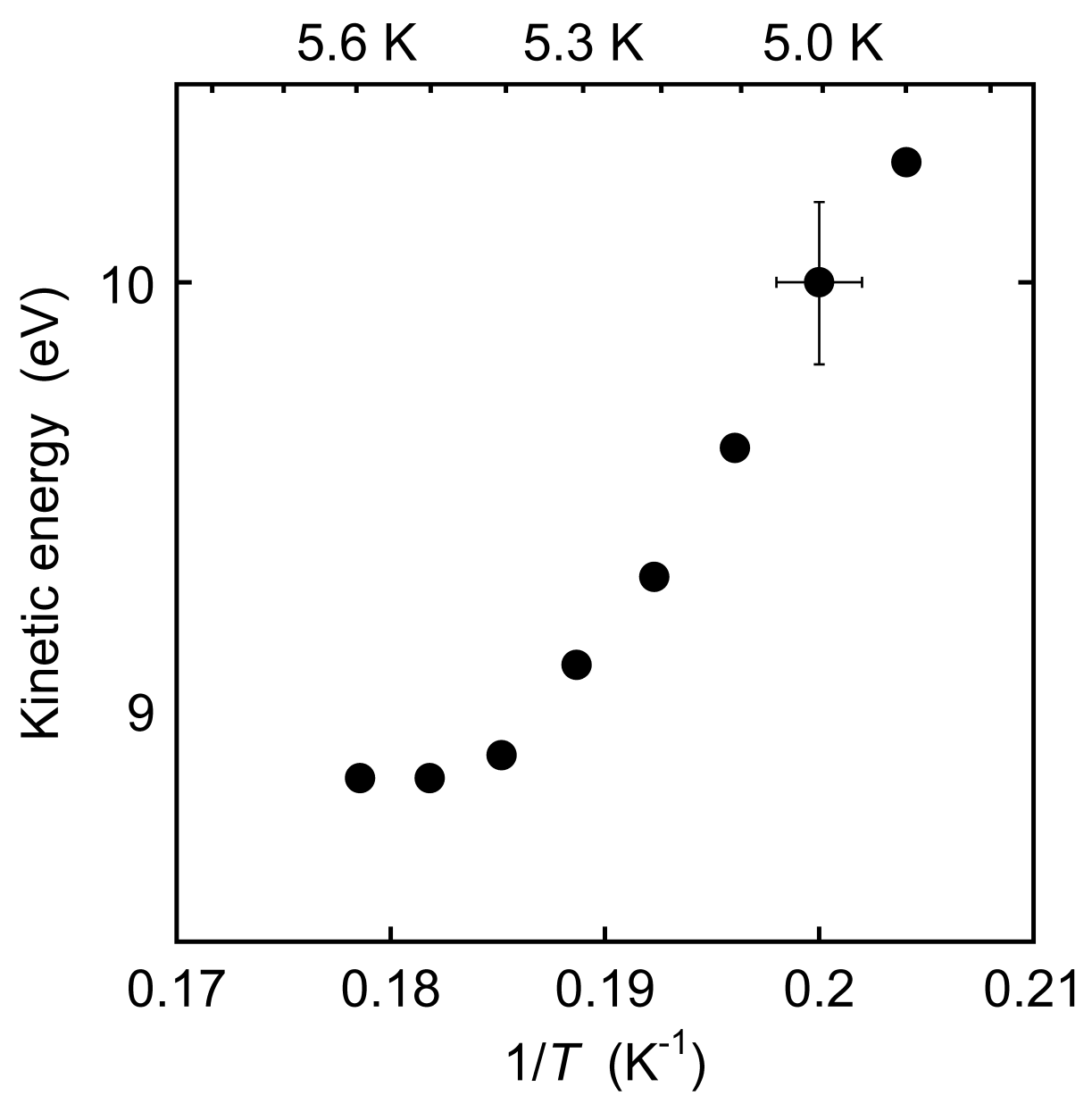

JINNO FIGURE. 5 